\title{
A NEW METHOD FOR DESIGNING LINEAR-PHASE RECOMBINATION NONUNIFORM FILTER-BANKS
}

\author{
X. M. Xie and S. C. Chan \\ Department of Electrical and Electronic Engineering \\ University of Hong Kong, Pokfulam Road, Hong Kong \\ \{xmxie, scchan\}@eee.hku.hk
}

\begin{abstract}
This paper proposes a new design method of linearphase (LP) recombination nonuniform filter-banks (RNFBs), where certain channels of a uniform filter-bank (FB) are merged by sets of transmultiplexers (TMUXs). The case where the numbers of channels of the uniform FB and the TMUXs are not coprime to each other is studied in detail. By analyzing the spectrum supports of the analysis filters, it is found that the uniform FB and recombination TMUXs in the LP RNFBs can be designed separately as long as certain conditions are satisfied. This significantly simplifies the design procedure. Using this result, the design of a class of nearly PR LP RNFBs with cosine roll-off transition band based on the REMEZ algorithm is studied. Design examples show that LP RNFBs with good frequency responses and reasonably low reconstruction error can be obtained readily by the proposed method.
\end{abstract}

\section{Introduction}

The theory and design of uniform perfect recombination (PR) filter banks (FBs) has been widely studied. In certain applications such as image, audio and speech analysis and coding, PR FBs with nonuniform frequency spacing usually offer considerable flexibility in frequency partitioning. This has attracted considerable interests in designing nonuniform FBs [1$5,8-10]$. The direct method studied in [1] is an attractive method because the analysis filters of the FB are linear time invariant (LTI) and it has more control on the frequency characteristics. On the other hand, it also leads to considerable number of parameters and constraints in the nonlinear optimization, which makes the design very involved. Another useful approach is to merge certain channels of a uniform FB with sets of transmultiplexers (TMUXs) having smaller numbers of channels [2]. This is usually referred to as the indirect or recombination method or recombination nonuniform $\mathrm{FB}$ (RNFB). It was also observed in [3] and [4] that RNFB can achieve PR if the uniform FB and the recombination TMUXs are $\mathrm{PR}$. In addition, by using cosinemodulated filter banks (CMFB) in this structure, recombination nonuniform CMFB (RN-CMFB) with very good frequency response can be designed readily. It was also shown in [3] that if the numbers of channels of the uniform FB and recombination TMUX are coprime, then the analysis filters of the resulting RNFB admit an equivalent LTI filter representation. The design procedure is therefore largely simplified. In [4], the more general case without the coprime assumption was studied. It was shown that although the analysis/synthesis filters are linear periodic time varying (LPTV), by imposing simple condition on the prototype filters, RN-CMFBs with very good frequency characteristics can still be achieved. The method proposed in [5] is also based on the merging approach, where a simple transformation is employed instead of the recombination TMUX. This method is useful in coding application, where partial spectrum reconstruction or good frequency characteristic is not required. In the latter cases, general recombination TMUXs have to be used.

In this paper, we propose a simple design method for a class of RNFBs with LP analysis/synthesis filters. We focus on the case where the channel numbers of the uniform and recombination TMUXs are not coprime to each other. In other words, the analysis filters are LPTV. By analyzing the spectrum supports of the analysis filters, it is found that the uniform FB and recombination TMUXs in the LP RNFBs can be designed separately as long as certain conditions are satisfied. Thus, the design can be significantly simplified. Using this result, we then focus on the design of a class of nearly PR LP RNFBs with cosine roll-off transition band using the REMEZ algorithm. Design examples show that LP RNFB with good frequency responses and reasonably low reconstruction error can be achieved. The paper is organized as follows: the principle of RNFBs briefly reviewed in section II. The spectral supports of the LPTV analysis filters in the RNFB are analyzed in section III. The design of the LP RNFBs is addressed and the conditions for the LP RNFBs with good filter quality are derived in Section IV. A design example is given in Section $\mathrm{V}$ and conclusions are drawn in section VI.

\section{Recombination Nonuniform Filter-Banks}

The structure of RNFBs is shown in Fig. 1, where sets of TMUXs with different numbers of channels are inserted in an $M$ channel uniform FB, yielding nonuniform subbands. For simplicity, only one TMUX with $m_{l}$ channels are plotted, where $l=0, \ldots, L-1$. The resulting sampling rate of this subband is $m_{l} / M$. It has been shown in [3] and [4] that if the uniform FB and the TMUXs are PR, then the nonuniform FB would be PR, provide that certain delays caused by the inserted TMUXs are compensated in other branches. For a simple analysis purpose, we ignore the multiplication operation with constant $c_{0} \sim c_{M-1}$, which does not affect the PR condition. This structure permits a structural PR property, which simplifies the design of the nonuniform FB. It is also shown in [3] that if $M$ and $m_{l}$ are coprime, this system allows an LTI representation of the analysis/synthesis filters like the direct structure in [1]. The RNFB has the advantage of lower implementation complexity and simple design. Another advantage is that the RNFBs can be achieved with larger range of sampling factors than that in the direct structure.

In the more general case where the numbers of channels of the FB and the TMUX are noncoprime, the analysis/synthesis filters of the RNFB are LPTV, though the entire system can achieve PR. The design of such LPTV system is therefore rather involved. The problem was considered in [4], where a method was proposed to minimize the undesirable components in the bifrequency responses of the analysis filters.

To begin with the analysis of the RNFB, let us consider the merged output in the RNFB. The discrete-time Fourier transform (DTFT) of the $l$-th merged output can be expressed as the following,

$$
Y_{l}\left(e^{j \omega}\right)=\frac{1}{M} \sum_{i=0}^{m_{j}-1} G_{l . i}\left(e^{j \omega}\right) \sum_{v=0}^{M-1} H_{n_{i+i}}\left(e^{j \frac{m_{l} \omega-2 \pi}{M}}\right) X\left(e^{j \frac{m_{l} \omega-2 \pi}{M}}\right),
$$

where $X\left(e^{j \omega}\right)$ and $Y_{i}\left(e^{j \omega}\right)$ are respectively the DTFT of the input and the $l$-th nonuniform subband output. Here, we assume that the $m_{l}$ channels are merged from the $r_{i}$-th channel of the $M$-channel uniform FB. The synthesis filters $G_{l, i}(\omega)$ of the $m_{l}$ channel TMUX is derived from an 1-skewed FB with synthesis 
filter $G_{i, i-F B}(\omega)$. So we have $G_{l, i}(\omega)=e^{-j \omega} G_{l, i-F B}(\omega)$. For notational convenience, we shall use $H(\omega)$ to denote $H\left(e^{j \omega}\right)$. Thus, (1) can be rewritten as

$$
Y_{t}(\omega)=\frac{1}{M} \sum_{v=0}^{M-1} A_{v}(\omega) X\left(\frac{m_{\ell} \omega-2 \pi v}{M}\right)
$$

where

$$
A_{v}(\omega)=\sum_{i=0}^{m_{t}-1} G_{l, i}(\omega) H_{r_{i}+i}\left(\frac{m_{, \omega} \omega-2 \pi v}{M}\right), \quad v=0,1, \cdots, M-1 .
$$

Note, $Y_{l}(\omega)$ has a period of $2 \pi \mathrm{M} / \mathrm{m}_{l}$. We would like to design $A_{v}(\omega)$ so that only the appropriate frequency components of $X(\omega)$ are extracted. Define $\bar{H}_{r_{+}+i}(\omega)=H_{r_{i}+i}\left(\frac{m_{i}}{M} \omega\right)$. Equation (3) can be rewritten as

$$
A_{v}(\omega)=e^{-j \omega} \sum_{i=0}^{m_{l}-1} G_{l, i-F B}(\omega) \bar{H}_{r_{i}+i}\left(\omega-2 \pi v / m_{l}\right) .
$$

Our objective is to identify the conditions on $\bar{H}_{r_{1}+i}(\omega)$ and $G_{l, i-F B}(\omega)$ such that $A_{v}(\omega)$ will possess the desirable frequency characteristics.

\section{LPTV Analysis Filters}

$$
\begin{gathered}
\bar{H}_{r_{i}+i}(\omega) \text { and } G_{l, i-F B}(\omega) \text { may be written as } \\
\bar{H}_{r_{i}+i}(\omega)=\bar{H}_{r_{i}+i}^{+}(\omega)+\bar{H}_{r_{i+i}}(\omega), \\
G_{l, i-F B}(\omega)=G_{i}(\omega)=G_{i}^{+}(\omega)+G_{i}^{-}(\omega),
\end{gathered}
$$

where $\bar{H}_{r_{i}+i}^{+}(\omega)$ and $\bar{H}_{r_{i}+i}^{-}(\omega)$ correspond to the responses of $\bar{H}_{r_{l}+i}(\omega)$ for positive and negative values of $\omega$ in $\left[-\frac{M}{m_{i}} \pi, \frac{M}{m_{l}} \pi\right]$, respectively. Since the periods of $H_{r_{i+i}}(\omega)$ and $G_{l, i-F B}(\omega)$ are $2 \pi$, the period of $\bar{H}_{r_{i}+i}(\omega)$ is $2 \pi M / m_{l}$. Similarly, $G_{i}^{+}(\omega)$ and $G_{i}^{-}(\omega)$ correspond to the responses of $G_{i}(\omega)$ in $\left[-\frac{\pi}{M}, \frac{\pi}{M}\right]$. For simplicity, we drop the subscription of $G_{l, i-F B}(\omega)$ and denote it as $G_{i}(\omega)$.

In our analysis, we assume that the stopband cutoff frequencies of $H_{v_{i+i}}(\omega)$ and $G_{i}(\omega)$ lie within $\left[\left(\left(r_{i}+i\right) \frac{\pi}{M}-\frac{\pi}{2 M}\right),\left(\left(r_{i}+i+1\right) \frac{\pi}{M}+\frac{\pi}{2 M}\right)\right]$ and $\left[\left(i \frac{\pi}{m_{i}}-\frac{\pi}{2 m_{i}}\right),\left((i+1) \frac{\pi}{m_{i}}+\frac{\pi}{2 m_{i}}\right)\right]$, respectively, and the stopband attenuation is sufficiently high that it can be treated as zero outside the region. Hence, only adjacent channels overlap in their magnitude responses. Denote the frequency support of a function $Q(\omega)$ as $\operatorname{Supp}(Q)$, which implies that if $\omega \notin \operatorname{Supp}(Q)$, then $Q(\omega)=0$. It can be shown that

$$
\begin{aligned}
\operatorname{Supp} & \left.\bar{H}_{i_{i}+i}\left(\omega-\frac{2 \cdot \pi}{m_{l}}\right)\right)=\operatorname{Supp}\left(\bar{H}_{i,+i}^{+}\left(\omega-\frac{2 \cdot \pi}{m_{l}}\right)\right) \cup \operatorname{Supp}\left(\bar{H}_{i_{i}+i}^{-}\left(\omega-\frac{2 \cdot \pi}{m_{l}}\right)\right) \\
= & \left\{\left(2 v+r_{l}+i-\frac{1}{2}\right) \frac{\pi}{m_{l}},\left(2 v+r_{l}+i+\frac{3}{2}\right) \frac{\pi}{m_{l}}\right\} \\
& \cup\left\{\left(2 v-r_{l}-i-\frac{3}{2}\right) \frac{\pi}{m_{l}},\left(2 v+-r_{l}-i+\frac{1}{2}\right) \frac{\pi}{m_{l}}\right\},
\end{aligned}
$$

$\operatorname{Supp}\left(G_{i}(\omega)\right)=\operatorname{Supp}\left(G_{i}^{+}(\omega)\right) \cup \operatorname{Supp}\left(G_{i}^{-}(\omega)\right)$

$$
\begin{aligned}
= & \left\{\left[2 q \pi+\left(i-\frac{1}{2}\right) \frac{\pi}{m_{l}}, 2 q \pi+\left(i+\frac{3}{2}\right) \frac{\pi}{m_{l}}\right], q= \pm 0, \pm 1, \pm 2, \cdots\right\} \\
& \cup\left\{\left[2 q \pi-\left(i+\frac{3}{2}\right) \frac{\pi}{m_{l}}, 2 q \pi-\left(i-\frac{1}{2}\right) \frac{\pi}{m_{l}}\right], q= \pm 0, \pm 1, \pm 2, \cdots\right\},
\end{aligned}
$$

where $p$ and $q$ are integers. Since the period of $\bar{H}_{r_{i}+i}\left(\omega-\frac{2 v \pi}{m_{l}}\right)$ for each given $v$ is $2 \pi M / m_{l}$. Therefore, we need only focus on the region $-\left(\pi M / m_{l}\right) \leq \omega \leq\left(\pi M / m_{t}\right)$, where

$$
p=0 \text { and }|q| \leq\left\lfloor\frac{M}{m_{l}}\right\rfloor .
$$

Here, $\lfloor x\rfloor$ denotes the nearest integer less than or equal to $x$.

Substituting (5) into (4), we get $A_{\nu}(\omega)=e^{-j \omega} \sum_{j=1}^{4} D_{j}^{(v)}(\omega)$, where

$$
\begin{aligned}
& D_{1}^{(v)}(\omega)=\sum_{i=0}^{m_{I}-1} \bar{H}_{r_{i}+i}^{+}\left(\omega-\frac{2 \cdot \pi}{m_{l}}\right) G_{i}^{+}(\omega), \\
& D_{2}^{(\omega)}(\omega)=\sum_{i=0}^{m_{i}-1} \bar{H}_{r_{i}+i}^{+}\left(\omega-\frac{2 \cdot \pi}{m_{l}}\right) G_{i}^{-}(\omega) ; \\
& D_{3}^{(v)}(\omega)=\sum_{i=0}^{m_{i}-1} \bar{H}_{r_{i+1}}^{-}\left(\omega-\frac{2 v \pi}{m_{i}}\right) G_{i}^{+}(\omega), \\
& D_{4}^{(v)}(\omega)=\sum_{i=0}^{m_{i}-1} \bar{H}_{r_{i}+i}^{-}\left(\omega-\frac{2 v \pi}{m_{t}}\right) G_{i}^{-}(\omega) .
\end{aligned}
$$

We just need to consider $D_{1}^{(v)}(\omega)$ and $D_{2}^{(v)}(\omega)$ since $D_{3}^{(v)}(\omega)$ and $D_{4}^{(v)}(\omega)$ are their conjugates.

The following conclusion can be derived in [8]. When $v \neq q m_{l}-r_{l}, D_{1}^{(v)}(\omega)=D_{2}^{(v)}(\omega)=0$. This means that the gain for the aliasing term is zero. When $v=q m_{l}-r_{l}, D_{1}^{(\nu)}(\omega)$ constitutes the passband of the LPTV filter $A_{v}(\omega)$ and $D_{2}^{(*)}(\omega)$ constitutes its stopband. Further if a so-called cross-term in $D_{2}^{(v)}(\omega)$ is cancelled the stopband attenuation can be ideal.

\section{LPTV Analysis Filter with LP Property}

For an LP filter $H\left(e^{j \omega}\right)$ with a symmetric (antisymmetric) impulse response $h(n)$, then we say $H\left(e^{j \omega}\right)$ is symmetric (anti-symmetric). If $\bar{H}_{r_{i}+i}(\omega)$ and $G_{i}(\omega)$ are symmetric, we have,

$$
\begin{aligned}
& \bar{H}_{r_{i}+i}^{+}(\omega)=e^{-j \alpha \omega}\left|\bar{H}_{r_{i+i}}^{R}(\omega)\right|, G_{i}^{+}(\omega)=e^{-j \beta \omega}\left|G_{i}^{R}(\omega)\right|, \\
& \bar{H}_{r_{i}+i}^{-}(\omega)=e^{-j \alpha \omega}\left|\bar{H}_{r_{i}+i}^{R}(\omega)\right|, G_{i}^{-}(\omega)=e^{-j \beta \omega}\left|G_{i}^{R}(\omega)\right| .
\end{aligned}
$$

Similarly, if $\bar{H}_{r_{i+i}}(\omega)$ and $G_{i}(\omega)$ are anti-symmetric, we have,

$$
\begin{aligned}
& \bar{H}_{r_{i}+i}^{+}(\omega)=j e^{-j \alpha \omega}\left|\bar{H}_{r_{i}+i}^{R}(\omega)\right|, G_{i}^{+}(\omega)=j e^{-j \beta \omega}\left|G_{i}^{R}(\omega)\right|, \\
& \bar{H}_{r_{i}+i}^{-}(\omega)=-j e^{-j \alpha \omega \mid}\left|\bar{H}_{r_{i}+i}^{R}(\omega)\right|, G_{i}^{-}(\omega)=-j e^{-j \beta \omega}\left|G_{i}^{R}(\omega)\right| .
\end{aligned}
$$

Here, $\bar{H}_{r_{i}+i}^{R}(\omega)$ and $G_{i}^{R}(\omega)$ are the amplitude responses, $\alpha=m_{l} N_{M} / 2$ and $\beta=M N_{m_{l}} / 2 . N_{M}$ and $N_{m_{l}}$ are the filter lengths of the FB and the TMUX, respectively.

In our study, two types of LP FBs are considered: filters with all symmetric property (same symmetry) and filters with alternative symmetric and anti-symmetric property (alternate symmetry). For the second type, we assume that $\bar{H}_{\eta_{i}+i}(\omega)$ and $G_{i}(\omega)$ are symmetric when $i$ is even, and anti-symmetric when $i$ is odd.

We now investigate $D_{1}^{(1)}(\omega)$ and $D_{2}^{(1)}(\omega)$ given in (8) with consideration of the constant $c_{r_{i}+i}$, which is used to achieve LP RNFB with good frequency characteristics. By using (9a) and $(9 \mathrm{~b}), D_{1}^{(1)}(\omega)$ and $D_{2}^{(())}(\omega)$ can be expressed as 


$$
\begin{aligned}
& D_{1}^{(\nu)}(\omega)=\sum_{i=0}^{m_{1}-1} c_{r_{1}+i} \bar{H}_{r_{i}+i}^{+}\left(\omega-\frac{2 v \pi}{m_{i}}\right) G_{i}^{+}(\omega)
\end{aligned}
$$

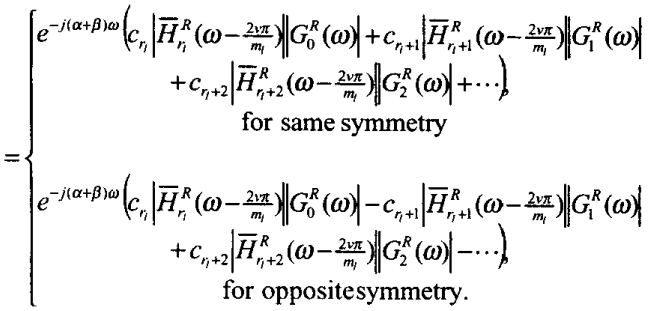

$$
\begin{aligned}
& D_{2}^{(v)}(\omega)=\sum_{i=0}^{m_{i}-1} c_{r_{i}+i} \bar{H}_{r_{i}+i}^{+}\left(\omega-\frac{2 v \pi}{m_{i}}\right) G_{i}^{-}(\omega) \\
& =\sum_{i=0}^{m_{i}-1} c_{r_{i+i}}\left(\bar{H}_{r_{i+i}}^{+}\left(\omega-\frac{2 v \pi}{m_{l}}\right) G_{i}^{-}(\omega) E_{F_{i}^{+}}+\bar{H}_{r_{i}+i}^{+}\left(\omega-\frac{2 v \pi}{m_{i}}\right) G_{i}^{-}(\omega) E_{F_{i}^{-}}\right) \\
& =c_{r_{i}} \bar{H}_{r_{i}}^{+}\left(\omega-\frac{2 v \pi}{m_{l}}\right) G_{0}^{-}(\omega) E_{F_{i}^{+}} \\
& +\sum_{i=0}^{m_{i}-2}\left(c_{r_{i+i+1}} \bar{H}_{r_{i}+i+1}^{+}\left(\omega-\frac{2 v \pi}{m_{i}}\right) G_{i_{i+1}}(\omega) E_{F_{i+1}}+c_{r_{i+i}} \bar{H}_{r_{i}+i}^{+}\left(\omega-\frac{2 v \pi}{m_{i}}\right) G_{i}^{-}(\omega) E_{F_{i}}\right) \\
& +c_{r_{i}+m_{i}-1} \bar{H}_{r_{i}+m_{l-1}}^{+}\left(\omega-\frac{2 v \pi}{m_{i}}\right) G_{m_{i}-1}^{-}(\omega) E_{F_{m_{i}-i}^{-}} .
\end{aligned}
$$

Note that there are two such supports in $D_{2}^{(v)}(\omega): \bar{H}_{r,+i}^{+}(\omega)$ and $G_{i}^{-}(\omega)$ overlapping at the left side of $\bar{H}_{r_{i}+i}^{+}(\omega)$ and at the right side of $\bar{H}_{r_{i}+i}^{+}(\omega)$. They are denoted by $F_{i}^{+}$and $F_{i}^{-}$, each having a length of $\pi / m_{l}$. Further, it can be shown that $F_{i+1}^{+}=F_{i}^{-}$. By introducing the indicator function of $F$ as $E_{F}$, i.e. $E_{F}(\omega)=\left\{\begin{array}{ll}1, & \text { if } \omega \in F \\ 0, & \text { if } \omega \notin F\end{array}\right.$, we have $E_{F_{i+1}^{+}}=E_{F_{i}^{-}}$. The term inside the large bracket (.) in (11) is the "cross-term", which is the source of the spurious response. It causes bumpings in the stopband of $A_{v}(\omega)$, which was first observed in [4] for RNCMFB. The cross-term can be written as

$$
\begin{aligned}
& c_{r_{i}+i} \bar{H}_{r_{i}+i}^{+}\left(\omega-\frac{2 v \pi}{m_{i}}\right) G_{i}^{-}(\omega) E_{F_{i}^{-}}+c_{r_{i}+i+1} \bar{H}_{r_{i}+i+1}^{+}\left(\omega-\frac{2 v \pi}{m_{i}}\right) G_{i+1}^{-}(\omega) E_{F_{i+1}^{+}} \\
& =e^{-j(\alpha+\beta) \omega}\left(c_{r_{i}+i}\left|H_{r_{i}+i}^{R}\left(\omega-\frac{2 v \pi}{m_{i}}\right)\right|\left|G_{i}^{R}(\omega)\right|\right. \\
& \left.\quad+c_{r_{i}+i+1}\left|H_{r_{i}+i+1}^{R}\left(\omega-\frac{2 v \pi}{m_{i}}\right)\right| G_{i+1}^{R}(\omega) \mid\right),
\end{aligned}
$$

for both same and alternate symmetries.

As mentioned earlier, $D_{1}^{(v)}(\omega)$ constitutes the passband of $A_{v}(\omega)$ and $D_{2}^{(v)}(\omega)$ constitutes its stopband. For a good filter, the passband need to be flat and stopband need to have high attenuation. Considering (11) and (12), we conclude that the requirement of passband flatness and ideal stopband attenuation may be satisfied by properly choosing the constant $c_{i_{i}+i}$ as shown in (13) and (14),

$$
\left\{\begin{array}{c}
c_{r_{i}+i}=c_{r_{i}+i+1}, \quad \text { for same symmetry } \\
c_{r_{i}+i}=-c_{r_{i}+i+1}, \quad \text { for alternativ e symmetry, } \\
c_{r_{i}+i}=-c_{r_{i}+i+1} .
\end{array}\right.
$$

Further, from (13) and (14) we obtained an existence condition: the LP RNFB must have alternate symmetry and $c_{r_{i}+i}$ must satisfy (14). This is a necessary condition for LP LTI RNFBs to have good frequency characteristics.

In order to cancel the spurious response, which may appear in the stopband of the LPTV filter $A_{\mathrm{v}}(\omega)$, the cross-term in (12) should be forced to zero. To this end, we get a matching condition: $\quad \bar{H}_{r_{i}+i}(\omega)=G_{i}(\omega), \quad$ or $\quad$ equivalently $H_{r_{i}+i}\left(\frac{m_{i}}{M} \omega\right)=G_{i}(\omega)$. The proof for a similar analysis using the RN-CMFB can be found in [8]. Due to space limitation, it is omitted here.

\section{Design Example}

An LP RNFB with decimation factors $(3 / 6,1 / 6,2 / 6)$ is designed. It is constructed by merging the first 3 channels and the last 2 channels of an 6-channel uniform LP FB with an 3-channel and an 2-channel LP TMUXs, respectively. The original FB and the recombination TMUXs can be designed separately as long as they satisfy the existence condition and the matching condition above. For the current example, the uniform LP FBs have equal filter lengths. The filters have alternate symmetry. For simplicity, we choose $c_{r_{i}+i}=1$ for even $i$ and $c_{r_{i}+i}=-1$ for odd $i$.

In the design of the uniform FB, we employ the REMEZ algorithm in MATLAB. The LP FB in our work is different from the LP paraunitary FB in [6] and the LP FBs by cosine and sine modulation in [7]. From the following the design procedure, we will see that the design method is very simple and the LP filters have ideal-bandwidth. In the design of 6-channel original uniform LP FB, the passband and stopband cutoff frequencies of the analysis filters are set to be: $H_{0}(z): \omega_{p_{0}}=0.4 \times \frac{2 \pi}{6}$, $\omega_{s_{0}}=0.6 \times \frac{2 \pi}{6} ; \quad H_{1}(z): \quad \omega_{s_{1}}=0.4 \times \frac{2 \pi}{6}, \quad \omega_{p_{1}}=0.6 \times \frac{2 \pi}{6}$, $\omega_{p_{2}}=0.9 \times \frac{2 \pi}{6}$ and $\omega_{s_{3}}=1.1 \times \frac{2 \pi}{6}$. The cutoff frequencies of the other analysis filters can are similarly defined. Here, $\omega_{p_{i}}$ and $\omega_{s_{i}}$ are respectively the passband and stopband cutoff frequencies of the filters. The relative weighting of the passband and stopband errors are chosen to be identical. The transition band of the analysis filters follows a cosine roll-off characteristic so that the FB is approximately power complementary and is nearly-PR. Because of this simplification, the desired responses of the analysis filters are completely determined and the maximum approximation error is minimized. The LP recombination TMUXs are designed under the above procedure. They are related to the original FB and satisfy the matching condition. The length of 6-channel LP FB is 126 and those of the 2-channel and 3-channel TMUXs are respectively 42 and 63 . This makes the matching condition much easier to be satisfied by setting $N_{M} / M=N_{m_{t}} / m_{l}$.

Since the channel numbers of the original FB and the recombination TMUX are not coprime, the analysis filter is LPTV. The bi-frequency responses of the LPTV analysis filter are illustrated in Fig. 2, where the amplitudes and phases of all the frequency components are generated by inputting a sinusoidal signal at certain frequency with unit amplitude and zero phase. In Fig. 2(a), the bi-frequency responses of the LPTV analysis filter with cutoff frequency at $\pi / 2$ are shown. This corresponds to the subband with sampling rate $(3 / 6)$ in the RNFB with decimation factors $(3 / 6,1 / 6,2 / 6)$. Fig. 2(b) shows their projection, by which we can easily observe the property of the LP analysis filter of the nonuniform FB. It can be seen that the LPTV filter has a good frequency response. In Fig. 2(c), the bi-frequency responses of the LPTV analysis filter with cutoff frequency at $2 \pi / 3$ corresponds to the subband with sampling rate $(2 / 6)$ in the RNFB with decimation factors $(3 / 6,1 / 6,2 / 6)$. Their projection in Fig. 2(d) shows the good quality of the LP LPTV analysis filter. In this system, the reconstruction and aliasing errors are $10^{-3}$.

From the above demonstration, it can be seen that the design of the proposed nearly-PR LP RNFBs is very simple and good filter quality can be achieved. 


\section{Conclusion}

A new method for designing LP RNFBs, where the numbers of channels of the uniform FB and the TMUXs are not coprime to each other is presented. An analysis of the spectrum supports of the analysis filters was performed and the condition when the uniform FB and recombination TMUXs in the LP RNFBs can be designed separately are derived. Using this result, the design of a class of nearly PR LP RNFBs with cosine roll-off transition band based on the REMEZ algorithm is studied. Design examples show that LP RNFBs with good frequency responses and reasonably low reconstruction error can be obtained readily by the proposed method.

\section{REFERENCES}

[1] J. Kovacevic and M. Vetterli, "Perfect reconstruction filter banks with rational sampling factors," IEEE Trans. Signal Processing, vol. 41, pp.2047-2066, Jun. 1993.

[2] R. V. Cox, "The design of uniformly and non-uniformly spaced pseudo quadrature mirror filters," IEEE Trans. Acoust., Speech, Signal Processing, vol. 24, pp. 1090-1096, Oct. 1986.
[3] S. C. Chan, X. M. Xie and T. I. Yuk, "Theory and design of a class of cosine-modulated non-uniform filter banks," in Proc. IEEE ICASSP, 2000, vol. 1, pp. 504-507.

[4] X. M. Xie, S. C. Chan and T. I. Yuk, "On the theory and design of a class of perfect-reconstruction nonuniform cosinemodulated filter-banks," in Proc. IEEE ISCAS, 2002, vol. 5, pp 285-288.

[5] O. A. Niamut and R. Heusdens, "Subband merging in cosinemodulated filter banks," IEEE Signal Processing Letters, vol. 10 pp. 111-114, Apr. 2003.

[6] A. K. Soman, P. P. Vaidyanathan, and T. Q. Nguyen, "Linearphase paraunitary filter banks: Theory, factorizations and applications," IEEE Trans. SP., Vol. 41, pp. 3480-3496, Dec. 1993.

[7] Y. P. Lin and P. P. Vaidyanathan, "Linear phase cosine modulated maximally decimated filter banks with perfect reconstruction," IEEE Trans. SP., Vol. 42, no.11, pp. 2525-2539, Nov. 1995.

[8] X M Xie "New design and realization methods for perfect reconstruction nonuniform filter banks," Ph.D. dissertation, The University of Hong Kong, Jan. 2004.

[9] P. Q. Hoang and P. P. Vaidyanathan, "Non-uniform multirate filter banks: theory and design," in Proc. IEEE ISCAS, 1989, pp. 371-374.

[10] S. J. Akkarakaran and P. P. Vaidyanathan, "New results and open problems on nonuniform filter-banks", Proc, ICASSP'99.

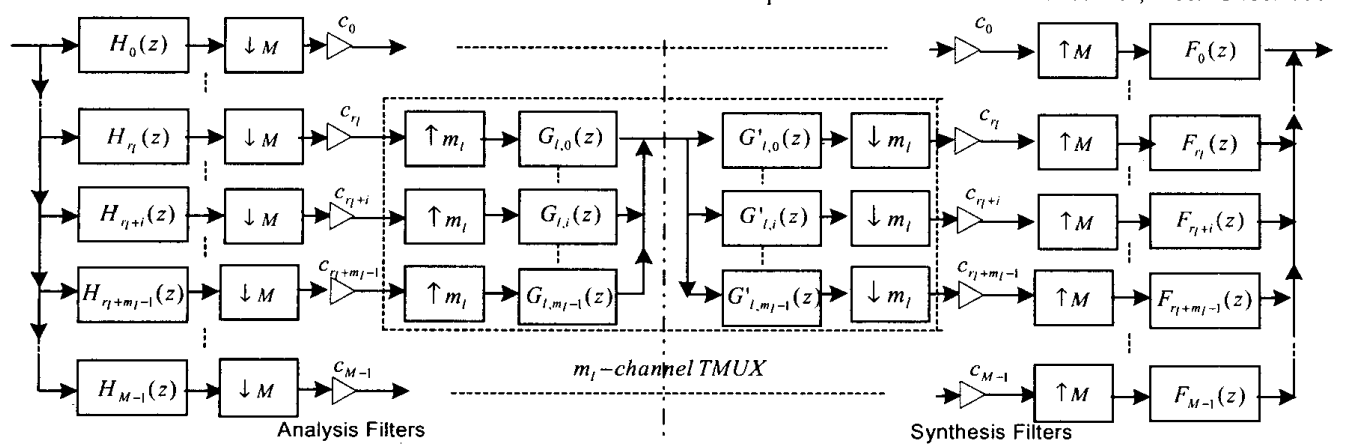

Fig. 1. Structure of RNFB

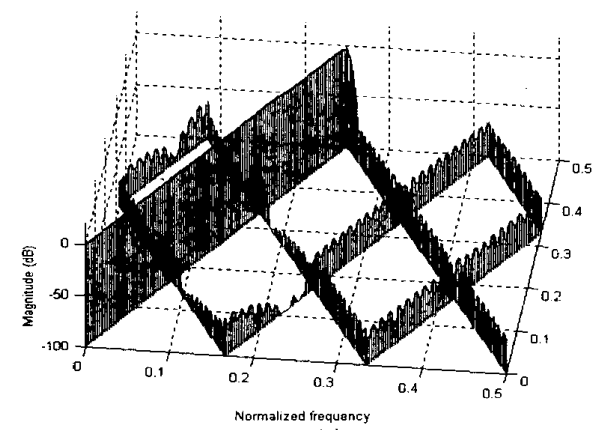

(a)

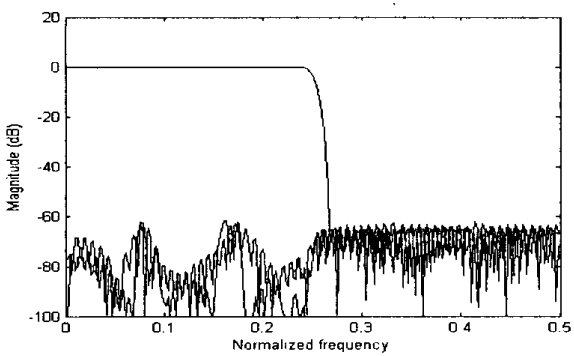

(b)

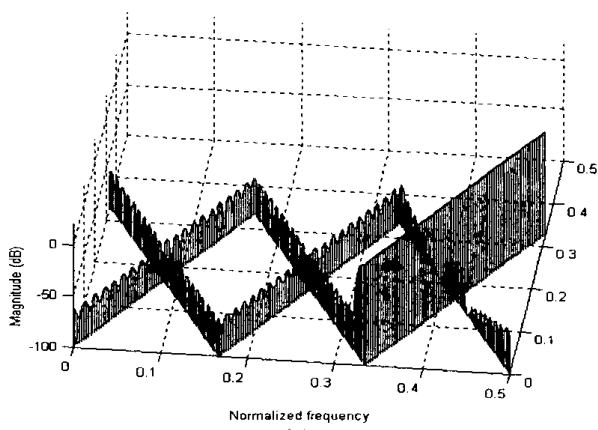

(c)

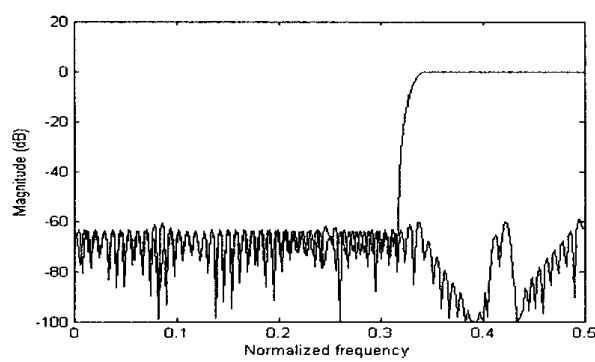

(d)

Fig. 2. Responses of the $1^{\text {st }}$ and $3^{\text {rd }}$ analysis filters in the LP RNFB with sampling rate $(3 / 6,1 / 6,2 / 6)$

(a) Bi-frequency responses of the $1^{\text {sa }}$ LPTV analysis filter and (b) their projection.

(c) Bi-frequency response of the $3^{\text {rd }}$ LPTV analysis filter and (d) their projection. 